Supporting Information

\title{
Dissecting the Stereocontrolled Conversion of Short-Lived Sulfenic Acid by Lachrymatory Factor Synthase
}

Takatoshi Arakawa, $,{ }^{1,2},{ }^{*}$ Yuta Sato, ${ }^{1}$ Masayuki Yamada, ${ }^{3}$ Jumpei Takabe, ${ }^{1}$ Yoshitaka Moriwaki, ${ }^{1,2}$ Noriya Masamura, ${ }^{5}$ Masahiro Kato, ${ }^{5}$ Morihiro Aoyagi, ${ }^{5}$ Takahiro Kamoi, ${ }^{5}$ Tohru Terada, ${ }^{2,4}$ Kentaro Shimizu, ${ }^{1,2}$ Nobuaki Tsuge, ${ }^{5}$ Shinsuke Imai, ${ }^{5}$ and Shinya Fushinobu ${ }^{1,2}$

${ }^{1}$ Graduate School of Agricultural and Life Sciences, ${ }^{2}$ Collaborative Research Institute for Innovative Microbiology, ${ }^{3}$ Graduate School of Information Science and Technology, ${ }^{4}$ Interfaculty Initiative in Information Studies, The University of Tokyo, Tokyo 113-8657, Japan. ${ }^{5}$ Basic Research Division, Central Research \& Development Institute, House Foods Group Incorporated, Yotsukaido 284-0033, Japan.

*Correspondence to: arakawa@mail.ecc.u-tokyo.ac.jp

\section{Table of Contents}

Experimental Methods $\quad$ S3

$\begin{array}{ll}\text { Computational Methods } & \text { S7 }\end{array}$

Figure S1. Comparison of crotyl alcohol-bound structures $\quad$ S11

Figure S2. Comparison of the present structures $\quad$ S12

Figure S3. Thermograms obtained from isothermal calorimetry measurements S13

$\begin{array}{llr}\text { Figure S4. Confirmation of the key residues } & \text { S14 }\end{array}$

Figure S5. Poses of 1-PSA in WT AcLFS in docking and MD simulations $\quad$ S15

Figure S6. Potential energy surface for the WT AcLFS-1-PSA system $\quad$ S16

Figure S7. Active site models of the Y114F variant at the stationary points $\quad$ S17

$\begin{array}{lll}\text { Figure S8. } & \text { MD simulation of a post-catalysis by AcLFS } & \text { S18 }\end{array}$

Figure S9. Potential energy scan for a PTSO molecule S19

$\begin{array}{lll}\text { Table S1. Data collection and refinement statistics } & \text { S20 }\end{array}$ 
Table S2. Primer sets used in this study

$\begin{array}{lll}\text { Table S3. Additional force field parameters for PTSO S23 } & \text { A }\end{array}$

References 


\section{Experimental Methods}

Construction of Plasmids. The sequences were amplified from a template plasmid harboring the complete open reading frame of $A c$ LFS (GenBank accession: AB089203). Fragments were digested with NdeI/XhoI and HindIII/BamHI, and then inserted into the corresponding sites of pET26b (Novagen) and pPAL7 (Bio-Rad) vectors to prepare the expression plasmids pET26b-AcLFS and pPAL7-AcLFS, respectively. Expression plasmids for $A c$ LFS mutants were prepared following the instructions of the PrimeStar mutagenesis kit (TaKaRa) using pET26b-AcLFS as the template. Primer sets used in this study are listed in Table $\mathbf{S 2}$.

Expression and Purification of $\boldsymbol{A c L F S}$. AcLFS and its variants were expressed in Escherichia coli BL21AI cells (Life Technologies). Cells were cultured at $37^{\circ} \mathrm{C}$ in $\mathrm{LB}$ medium containing $50 \mathrm{mg} / \mathrm{L}$ kanamycin. After $\mathrm{OD}_{600}$ reached $0.6, A c \mathrm{LFS}$ was induced for 2 hours by adding a final concentration of $1 \mathrm{mM}$ IPTG and $0.2 \% 1$ arabinose. Harvested cell pellets were suspended in solution A [50 mM Hepes-Na (pH7.5), $0.1 \mu \mathrm{m}$ PMSF] and disrupted by sonication (Sonifier 250D, Branson); then, insoluble particles were removed by centrifugation $(20,000 \times \mathrm{g}, 30 \mathrm{~min})$. The supernatants were loaded onto HisTrap FF crude $5 \mathrm{~mL}$ (GE Healthcare) equilibrated with solution B [50 $\mathrm{mM}$ Hepes- $\mathrm{Na}(\mathrm{pH} 7.5), 500 \mathrm{mM} \mathrm{NaCl}$ ] and the eluates for solution $\mathrm{C}$ (solution $\mathrm{B}$ containing $500 \mathrm{mM}$ imidazole) were pooled. The samples were loaded onto Superdex 200 10/300 GL (GE Healthcare) equilibrated with solution D [20 mM Hepes-Na (pH 7.5), $150 \mathrm{mM} \mathrm{NaCl}$. Peak fractions monitored by absorbance at $280 \mathrm{~nm}$ were collected and 
concentrated to around $20 \mathrm{mg} / \mathrm{mL}$ with the buffer exchanged by $20 \mathrm{mM}$ Hepes- $\mathrm{Na}(\mathrm{pH}$ 7.5) using Vivaspin turbo 10K MWCO (Sartorius).

For the production of selenomethionine (SeMet)-derivatized AcLFS, pPAL7-AcLFS was introduced into E. coli BL21 CodonPlus(DE3) RP-X cells. These cells were cultured in LeMaster medium containing $100 \mathrm{mg} / \mathrm{L}$ ampicillin and $35 \mathrm{mg} / \mathrm{L}$ chloramphenicol, and IPTG was added to the medium at a final concentration of $1 \mathrm{mM}$ for induction. Extraction and purification were performed as per the protocols of non-derivatized $A c \mathrm{LFS}$, except for using a Profinity eXact column (Bio-Rad) instead of a HisTrap FF crude column, in accordance with the manufacturer's instructions. In each step, purity was checked by SDS-PAGE and protein concentration was estimated using BCA protein assay kit (Thermo Fisher Scientific).

Crystallography and Structure Determination. Crystallization was performed by the sitting-drop vapor diffusion method at $20^{\circ} \mathrm{C}$. Crystals appeared within a week from the several batches of the conditions of the JCSG core I-IV and plus kits (Qiagen); then, the reservoir compositions were specifically optimized to the following condition: 20\% PEG6000, 0.1 M MES-Na (pH 6.0) for crotyl alcohol complex, and 2.0 $\mathrm{M}$ ammonium sulfate, $0.1 \mathrm{M}$ MES-Na ( $\mathrm{pH}$ 6.0) for the rest. Each single crystal on the drops was picked out on CryoLoop (Hampton Research) and directly snapped in liquid nitrogen after cryo-protection, which was performed by transferring the crystal gradually to the reservoir solution containing $20 \%$ (v/v) glycerol or $16 \%$ D-trehalose. Immersion of the crystals in 1,2-propanediol or crotyl alcohol [final 2\% (v/v) and $20 \mathrm{mM}$, respectively] was performed together with the D-trehalose treatment. 
Diffraction experiments were carried out at synchrotron facilities of KEK (Tsukuba, Japan), employing the mounting robot $\mathrm{PAM}^{1}$ and client software UGUIS. The data acquisition scheme was basically set as follows: exposure of a $100 \times 100 \mu \mathrm{m}^{2}$ beam for 1 sec per frame and the collection of 180 (360 for the SeMet dataset) frames with $1^{\circ}$ oscillation steps. Diffraction images were indexed, integrated, and scaled using the HKL2000 program suite (HKL Research Inc.) or XDS².

Initial phases were determined by the single-wavelength anomalous dispersion (SAD) method using the SeMet dataset, practically by using the phenix.autosol module implemented in Phenix program suite ${ }^{3}$. The resultant model was built mostly via phenix.autobuild and extended to the whole of the molecule manually, with viewing using the program $\mathrm{Coot}^{4}$. Initial models corresponding to the other datasets were determined by molecular replacement, using the program Molrep ${ }^{5}$ if needed. Models were refined iteratively using REFMAC5 in the CCP4 program suite ${ }^{6-7}$ and heteroatoms found were added according to $m\left|F_{\mathrm{o}}\right|-D\left|F_{\mathrm{c}}\right|$ maps at the posterior rounds. The crystals obtained from ammonium sulfate condition originally belong to the $P 2{ }_{1} 2{ }_{1} 2_{1}$ space group, but are prone to being adapted as $P 4_{1}$ during pretreatment processes. The twin refinement option implemented in REFMAC5 was applied for the datasets indexed as the $P 4_{1}$ space group since nearly perfect twinning was found by phenix.xtriage. In addition, the occupancies of all of the atoms were set to 0.8 in the REFMAC5 calculations to reduce the phase biasconducted lack of the resulting map densities around a region of one of the eight molecules in an asymmetric unit. The final model was verified by respective composite omit maps. Crystallographic and refinement statistics are listed in Table S1. Molecular 
graphics including superposition and alignments of models were prepared using PyMOL (Schrödinger LLC). Electrostatic potentials were calculated using $\mathrm{APBS}^{8}$ with the standard parameters. Coordinates and structure factors were deposited in the Protein Data Bank (PDB) with the accession codes of 5GTE (solute-free), 5GTF (with glycerol), 5GTG (with 1,2-propanediol), and 6IES (with crotyl alcohol).

Product Assay. Liberated PTSO was isolated by reverse-phase chromatography at $25^{\circ} \mathrm{C}$, as described previously ${ }^{9}$. Briefly, a 3-min-reaction mixture was subjected to Pegasil ODS $4.5 \times 250$ (Senshu Kagaku) equilibrated with $0.01 \%$ trifluoroacetic acid in 30\% methanol solution ( $\mathrm{pH} 3.3$ ) and then developed for $12 \mathrm{~min}$ with a flow rate of $0.6 \mathrm{~mL} / \mathrm{min}$. Absorbance at $254 \mathrm{~nm}$ was monitored using an Alliance instrument (Waters). A total batch of $25 \mu \mathrm{L}$ initially consisted of $5 \mu \mathrm{L}$ of $0.5 \mu \mathrm{M}(0.01$ $\mathrm{mg} / \mathrm{mL}) A c \mathrm{LFS}, 5 \mu \mathrm{L}$ of respective inhibitor in 5 -fold concentrate and $10 \mu \mathrm{L}$ of garlic alliinase $(250 \mathrm{U} / \mathrm{mL})$, then $5 \mu \mathrm{L}$ of $113 \mathrm{mM}(20 \mathrm{mg} / \mathrm{mL})$ trans-1-PRENCSO was added to start the reaction. Cysteine thiols on $A c$ LFS were inactivated by adding iodoacetamide at a final concentration of $1 \mathrm{mM}$ in $0.5 \mu \mathrm{M} A c$ LFS and incubating the mixture at $4^{\circ} \mathrm{C}$ for several hours in the dark. Chemical reagents were purchased from Wako Pure Chemicals and Tokyo Chemical Industry. Enzyme unit (U) of alliinase is defined as the activity producing $1 \mu \mathrm{mol}$ of pyruvic acid per $1 \mathrm{~min}$.

Isothermal Calorimetry. Experiments were performed at 20 and $25^{\circ} \mathrm{C}$ using Nano ITC SV (TA Instruments). Net heat were measured during addition of solute molecules (glycerol and crotyl alcohol) on a solid gold cell. The solution of purified AcLFS was exchanged with solution D and $100 \mu \mathrm{L}$ of $A c$ LFS was loaded on the cell. 
Titrants were prepared with dilution of respective ligands with the same solution. AcLFS solution was stirred at $350 \mathrm{rpm}$ in a $1.3 \mathrm{~mL}$ cell and titrated with $5 \mu \mathrm{L}$ of the ligand repeatedly at intervals of $60 \mathrm{sec}$. Ligand was titrated into buffer solution to compare the heat of dilution.

\section{Computational Methods}

\section{System Preparation, Equilibration, and Molecular Dynamics Simulation.}

The crystal structure of $A c$ LFS in glycerol-bound form (PDB ID: 5GTF) was used as the initial protein model of the molecular dynamics (MD) simulations after adding missing hydrogen atoms. The initial pose of 1-PSA was determined by superimposing its $\mathrm{C}_{2}, \mathrm{C}_{3}$, and $\mathrm{S}$ atoms of 1-PSA onto the $\mathrm{C}_{3}, \mathrm{C}_{2}$, and $\mathrm{C}_{1}$ atoms of glycerol, respectively.

The prepared structure was fully solvated in 13,370 molecules of the TIP3P explicit water mode ${ }^{10}$ in a cubic periodic box, and neutralized by adding eight $\mathrm{Na}^{+}$counter ions via the Amber LEaP module ${ }^{11}$. The ff14SB force field was used for the protein. The substrate was parameterized using the General AMBER force field (GAFF) ${ }^{12}$ as part of the antechamber package ${ }^{13}$ and restrained electrostatic potential (RESP) charges obtained using HF/6-31G(d) single-point calculations on the optimized geometries at the B3LYP/6-31G(d) level. During MD simulations for isolated PTSO, geometric isomerization of PTSO occurred around the $\mathrm{C}_{1}-\mathrm{S}$ bond when the parameters assigned by GAFF were used. Because the isomerization does not occur in nature ${ }^{14}$, we redefined the cosine function of the torsional angle $\mathrm{C}_{2}-\mathrm{C}_{1}-\mathrm{S}-\mathrm{O}$ by calculating the $\mathrm{QM}$ energies at the B3LYP/6-31+G(d,p) level of theory (Figure S9). It was implemented in the MD 
simulations, together with the additional torsional parameters shown in Table S3. Short range van der Waals and electrostatic interactions were cut off beyond $10 \AA$ and the particle mesh Ewald (PME) method ${ }^{15}$ was used for long-range interactions. The system was first relaxed using 200 steps of the steepest descent minimization with a 1,000 kcal $\mathrm{mol}^{-1} \AA^{-2}$ constraint applied to the heavy atoms of the protein. Subsequently, the constraint was removed, and the entire system was subjected to 200 steps of the steepest descent minimization. Next, to gradually heat the system, MD simulations over $1 \mathrm{~ns}$ in duration at a temperature of $300 \mathrm{~K}$ under $N P T$ ensemble were performed. Finally, five 200 ns MD simulations in the NPT ensemble representing the production run were carried out with snapshots saved every 10 ps. The Leap-frog algorithm with a time step of $2 \mathrm{fs}$ was used, and the $V$-rescale ${ }^{16}$ thermostat and Parrinello-Rahman barostat were used to control the temperature $(300 \mathrm{~K})$ and pressure $(1.00$ bar $)$ of the MD simulations, respectively.

ONIOM Computational Scheme. Three snapshots taken from each production run of the MD simulation were adopted, in which the $\mathrm{C}_{2}-\mathrm{O}_{\varepsilon 1}$ distance between 1-PSA and E88 was $3.19 \AA, 3.36 \AA$ and $3.53 \AA$, respectively. To generate our own $N$ layered integrated molecular orbital and molecular mechanics (ONIOM) models from these structures, water molecules more than $5.0 \AA$ away from the substrate or protein atom were removed. Subsequently, we applied a two-layer extrapolated ONIOM method to each structure ${ }^{17}$, in which the QM and MM layers were treated by the B3LYP ${ }^{18-19}$ method and AMBER force field, respectively. The QM layer consisted of side chains of R71, E88, Y102, Y114, and the bound 1-PSA. There were no water molecules that 
directly interact with any of these residues. The covalent boundary between the QM and MM layers was capped by hydrogen link atoms. The MM layer was further separated into two layers: (1) a mobile MM layer that could optimize and (2) a MM layer that was fixed to the original structure. The mobile MM layer consisted of all amino acids and water molecules within 7.0 $\AA$ of the QM layer atoms, whereas the fixed MM layer consisted of any protein or water atoms not present in the QM layer and the mobile MM layer. The MM layers were described with the ff14SB and GAFF.

To generate PES, B3LYP/6-31G(d) was initially used to describe the QM layer. The $\mathrm{O}^{\mathrm{b}}-\mathrm{H}^{\mathrm{b}}$ and $\mathrm{C}_{2}-\mathrm{H}^{\mathrm{b}}$ distances of the substrate were chosen as the reaction coordinates. To generate the grid points of energy calculations of our ONIOM scheme, the first coordinate was scanned using a step-size $0.27 \AA$ (from $1.013 \AA$ to $3.443 \AA$ ) and the second coordinate $-0.23 \AA$ (from $3.048 \AA$ to $0.978 \AA$ ). A coarse reaction surface was obtained by connecting total 100 energy values calculated from optimizations relaxing the entire geometry except for the $\mathrm{O}^{\mathrm{b}}-\mathrm{H}^{\mathrm{b}}$ and $\mathrm{C}_{2}-\mathrm{H}^{\mathrm{b}}$ distances assigned by the grid points.

Subsequently, to afford an accurate description of the active site and the energy values, we re-optimized the stationary points identified on the coarse reaction surfaces with ONIOM(B3LYP/6-31+G(d,p):AMBER) without constraints on the reaction coordinates. The procedure for fitting a consistent set of partial charges, which was developed by Tao et $a l^{20}$, was used to optimize all the equilibrium states and transition states. In the procedure, each geometry was first optimized using our ONIOM scheme. Subsequently, the RESP charges were recalculated for the QM layer using the electrostatic grid obtained from a gas-phase B3LYP/6-31+G(d,p) single-point calculation 
on the extracted QM layer with hydrogen atoms capping truncation points. Finally, we re-optimized the system using the new charges. This procedure was repeated until the difference in the total $\mathrm{QM} / \mathrm{MM}$ energies from the last two geometries was converged to less than $0.1 \mathrm{kcal} / \mathrm{mol}$. All the equilibrium states and transition states were optimized by the mechanical embedding (ME) scheme. For each transition state, frequency calculations were performed at the optimization level of theory. Intrinsic reaction coordinate (IRC) calculations were further conducted to verify the transition states. The electronic embedding (EE) scheme was adopted for single-point energy calculations on the ONIOM-ME optimized structures. All the energies presented in the text are the relative potential energies at $\mathrm{ONIOM}(\mathrm{B} 3 \mathrm{LYP} / 6-31+\mathrm{G}(\mathrm{d}, \mathrm{p})$ :AMBER $)$-EE level relative to the reactant state. Molecular orbitals were obtained from the ONIOM(B3LYP/6$31+\mathrm{G}(\mathrm{d}, \mathrm{p})$ :AMBER)-EE single-point calculations. Natural bond orbital (NBO) analyses were conducted for the QM layers of all equilibrium states and transition states using NBO version 3. All the ONIOM calculations were carried out with Gaussian 16 Rev. A. 03. 

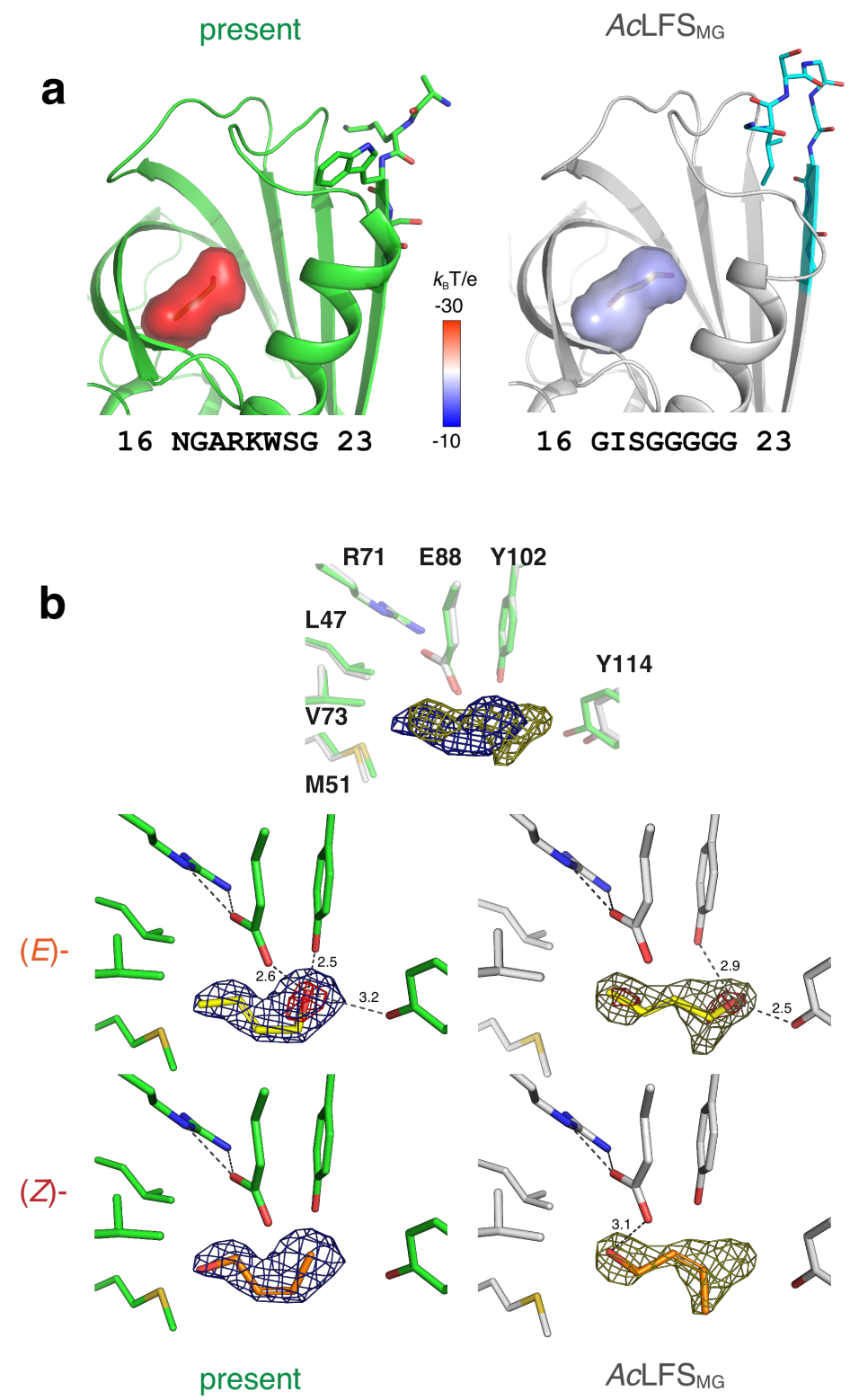

Figure S1. Comparison of crotyl alcohol-bound structures. (a) Schematic drawings of the present (left) and $A c \mathrm{LFS}_{\mathrm{MG}}$ (right) structures. Sticks represent the residues in the direction of each N-terminus identified by the crystallographic analyses. Amino-acid sequences from 16 to 23 are written below the panels. The surface represents the pocket inside $A c$ LFSs and are colored according to the electrostatic potentials calculated as in the neutral pH. (b) Assignment of crotyl alcohol in the map. Close-up views of structures

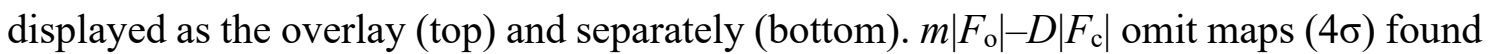
in $A c$ LFS and $A c$ LFS $_{\text {MG }}$ are displayed in blue and yellow meshes, respectively. Yellow sticks represent the assigned crotyl alcohol isomers. Dashes are the probable hydrogen bonds with distances. 
a

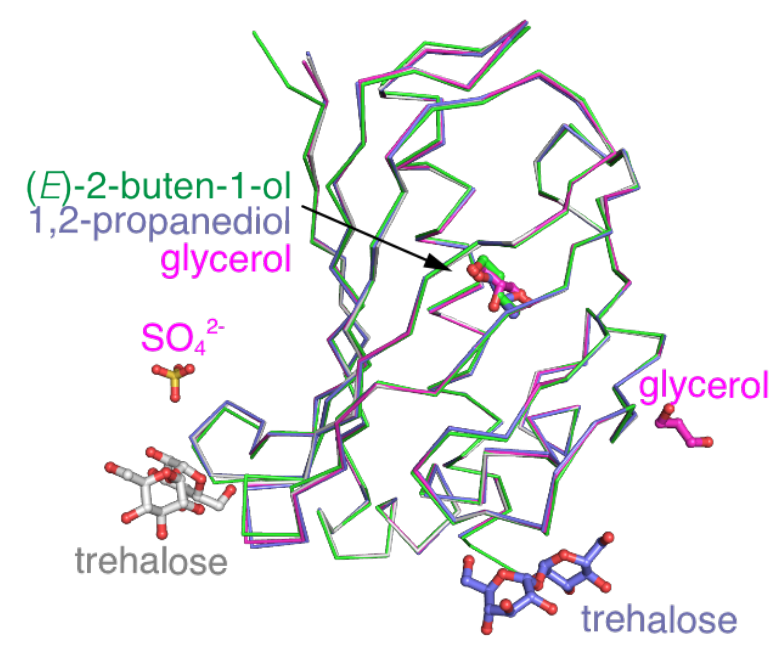

b
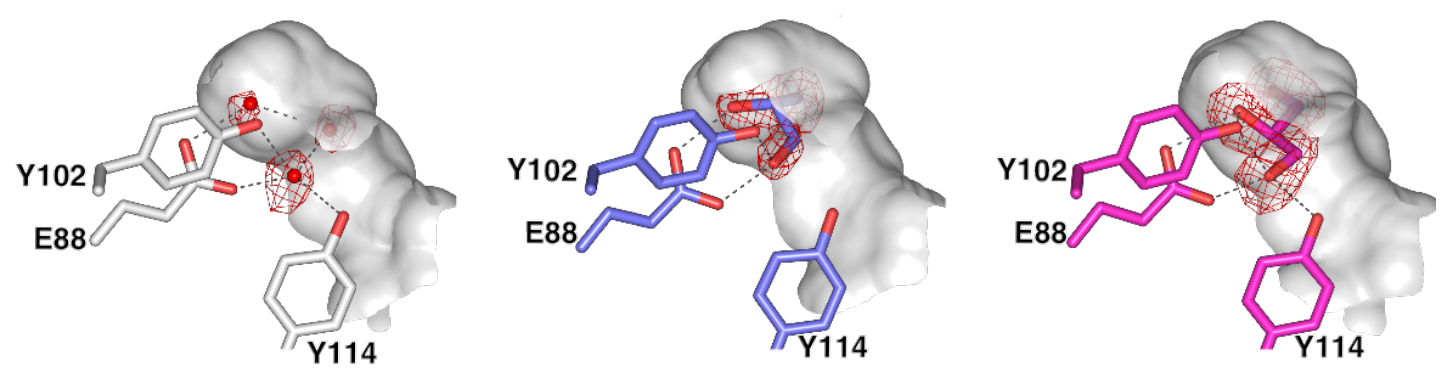

Figure S2. Comparison of the present structures. (a) Superposition of a protomer of each model. Traces of $\mathrm{C} \alpha$ atoms are taken from the data of +crotyl alcohol (green), +1,2propanediol (violet), +glycerol (magenta), and solute-free (gray). Sticks represent exogenous compounds observed on the crystals. (b) Non-protein electron densities found at the active site. $m\left|F_{\mathrm{o}}\right|-D\left|F_{\mathrm{c}}\right|$ omit maps $(3 \sigma)$ are displayed in red meshes. Surfaces colored in gray are provided by the hydrophobic residues. Dashes represent suggested hydrogen bond pairs. 
a

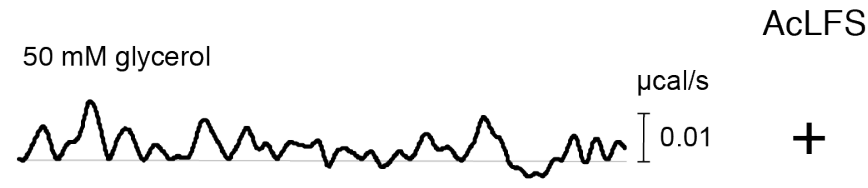

$20 \mathrm{mM}$ crotyl alcohol

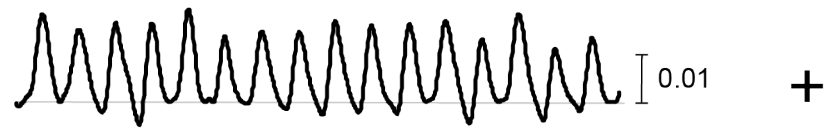

$20 \mathrm{mM}$ crotyl alcohol

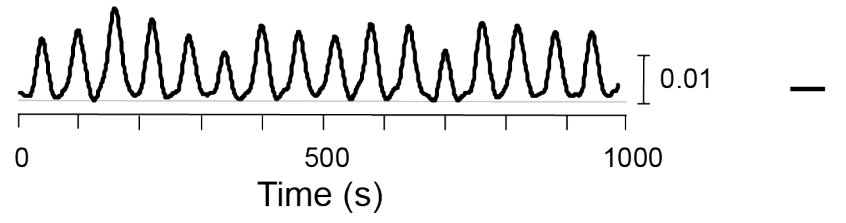

b

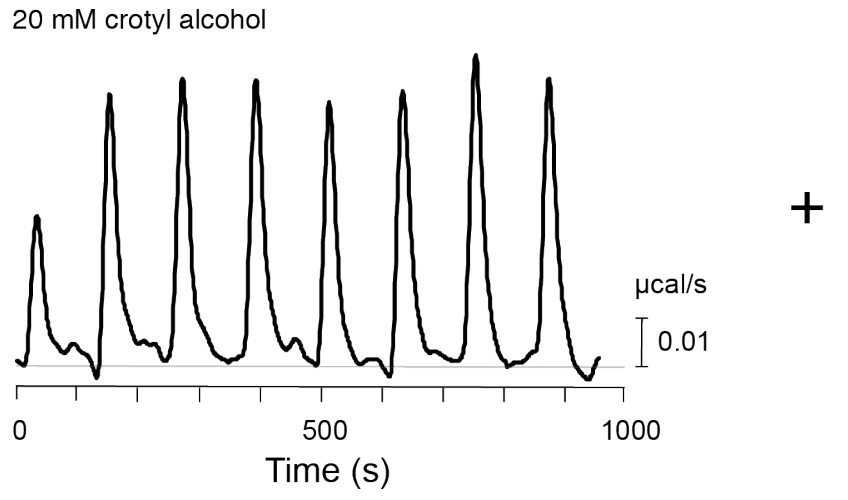

Figure S3. Thermograms obtained from isothermal calorimetry measurements. Experiments are done at $20^{\circ} \mathrm{C}$ (a) and $25^{\circ} \mathrm{C} \mathrm{(b)}$, and net heat expenses from iterated titration in the presence $(+)$ and absence (-) of $150 \mu \mathrm{M} A c \mathrm{LFS}$ are shown. Titrations using $50 \mathrm{mM}$ glycerol and $20 \mathrm{mM}$ crotyl alcohol are tested. 


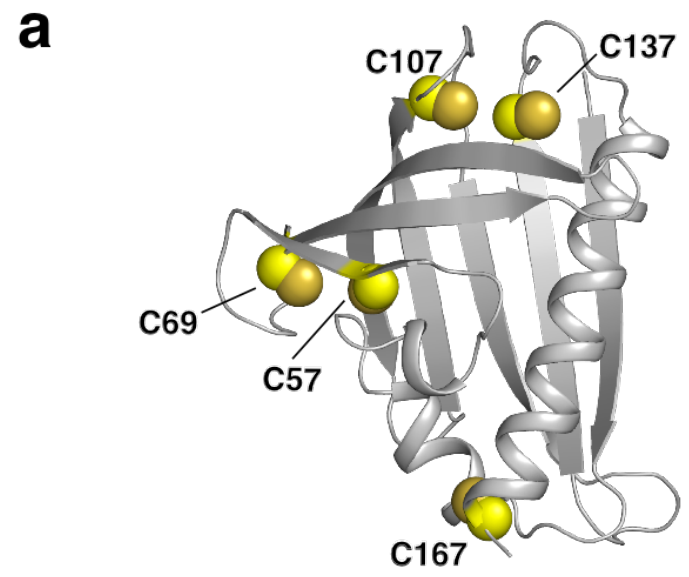

b

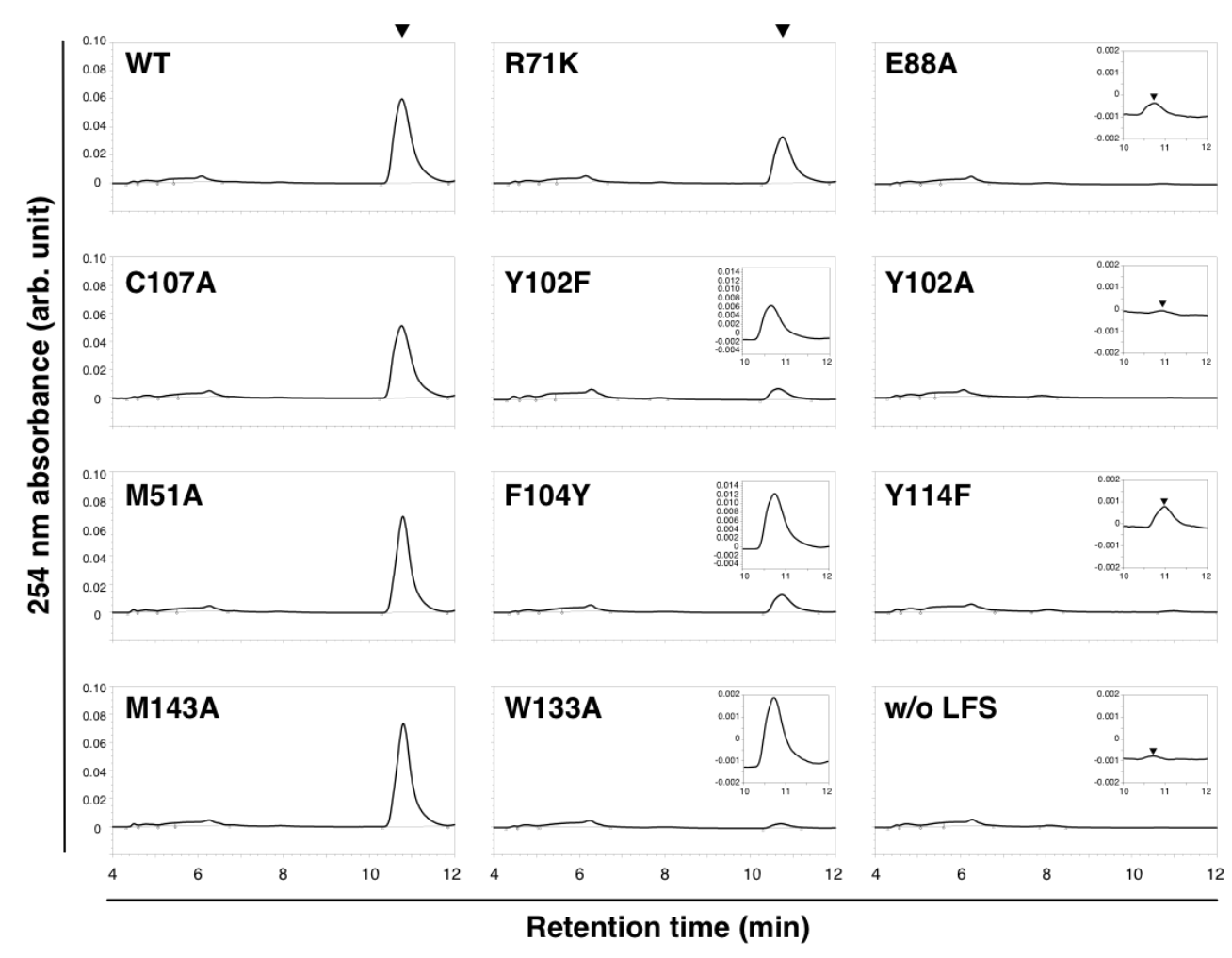

Figure S4. Confirmation of the key residues. (a) Distribution of cysteine residues on AcLFS structure. (b) Chromatograms of the selected measurements of reaction products. Arrowheads indicate the retention times of PTSO. 
a

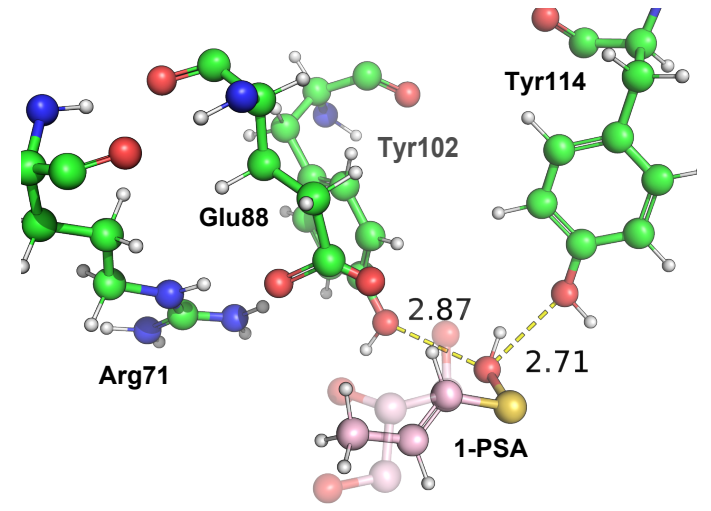

b

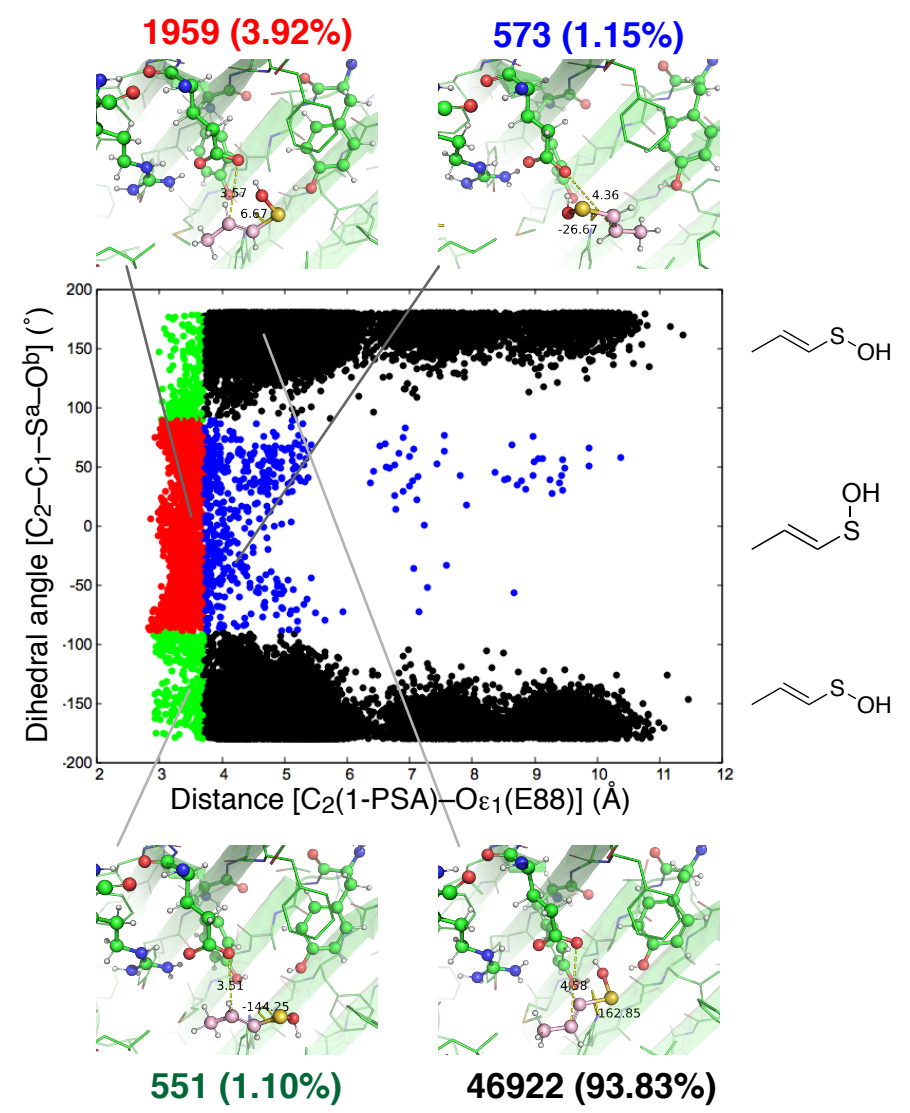

Figure S5. Poses of 1-PSA in AcLFS in docking and MD simulations. (a) Energyminimized active site model of WT AcLFS-1-PSA complex prepared from docking simulation. Glycerol used as the guide is overlaid. (b) Distribution plot of positions and molecular topologies of 1-PSA found in MD simulations. Samples are obtained from total $500 \mathrm{~ns}$ simulation and each point corresponds a 10 ps-run, colored by the classification according to dihedral angles around $\mathrm{C}_{1}-\mathrm{S}^{\mathrm{a}}$ bond (threshold angle $\pm 90^{\circ}$ ) and distances between $\mathrm{C}_{2,1-\mathrm{PSA}}$ and $\mathrm{O}_{\varepsilon 1, \mathrm{E} 88}(3.7 \AA)$. A typical snapshot of each class is presented with numbers of samples. 
a

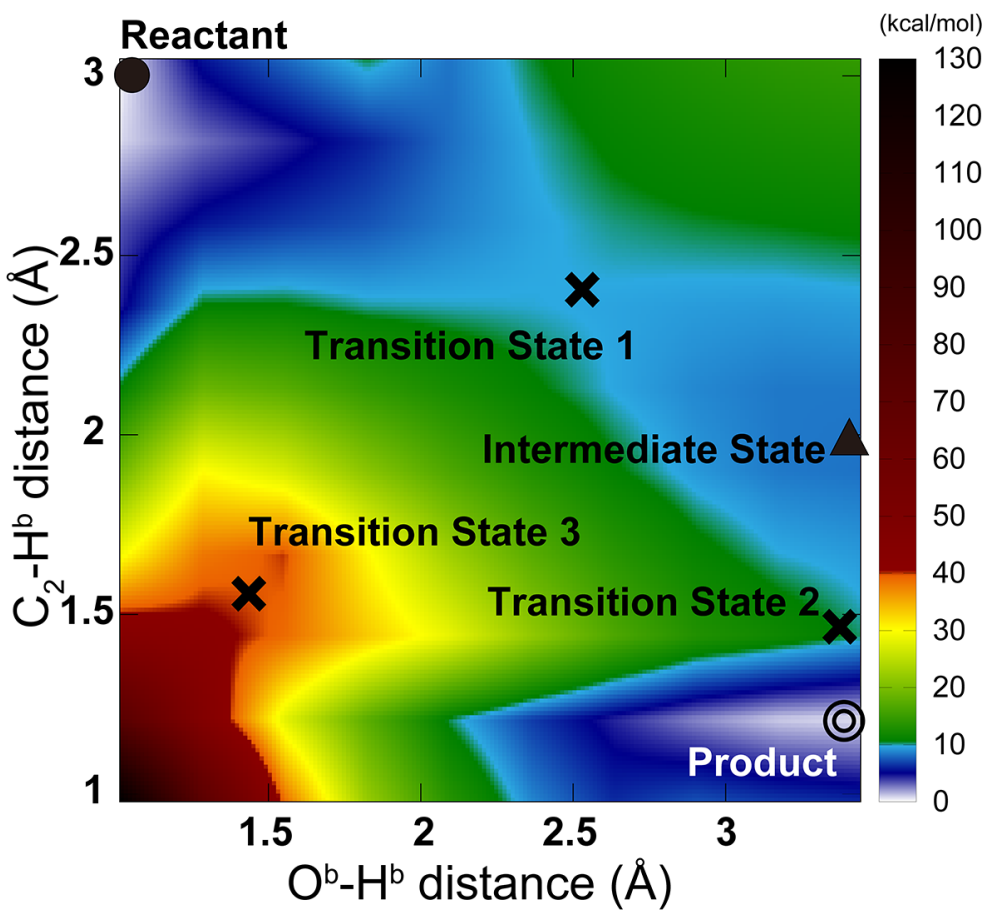

b

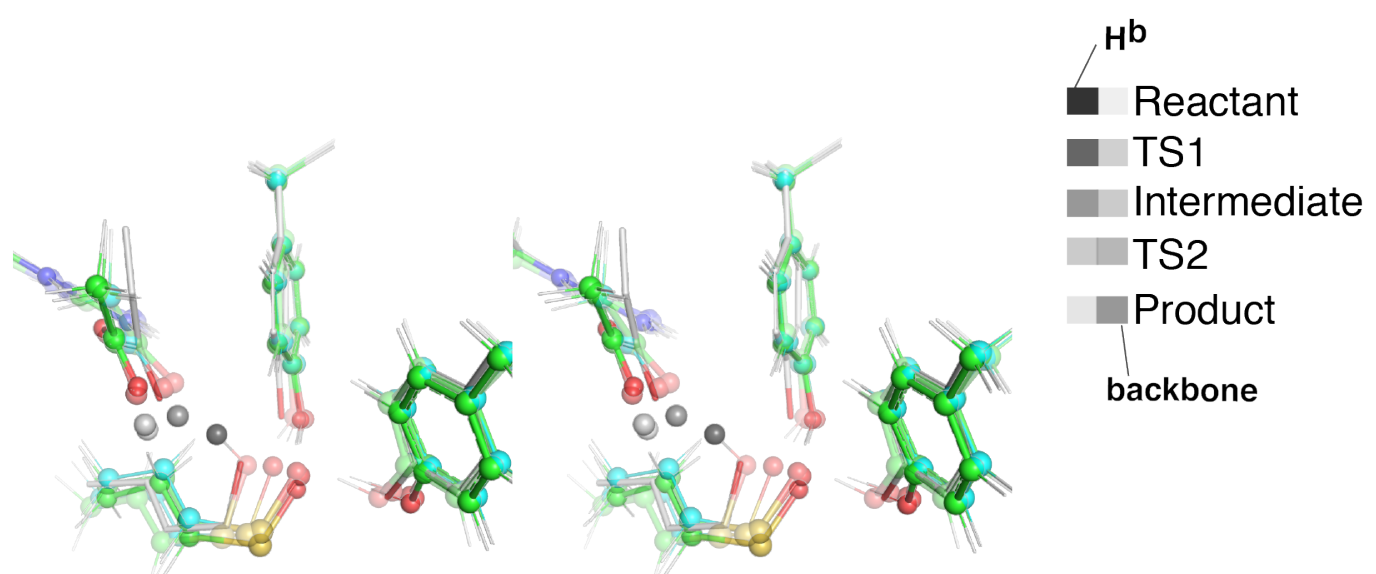

Figure S6. Potential energy surface for the WT AcLFS-1-PSA system. (a) The QM/MMbased energy profile drawn according to the two distances $\left(\mathrm{C}_{2}-\mathrm{H}^{\mathrm{b}}\right.$ and $\left.\mathrm{O}^{\mathrm{b}}-\mathrm{H}^{\mathrm{b}}\right)$ in the QM layer. Marked stationary points correspond to the reaction states in the given reaction pathways (see main text). Energies are shown in $\mathrm{kcal} / \mathrm{mol}$. (b) Stereoview of superimposition of stationary state structures along the plausible reaction pathway. R71, E88, Y102, Y114 and 1-PSA are shown. Gray spheres represent $\mathrm{H}^{\mathrm{b}}$ atoms. 


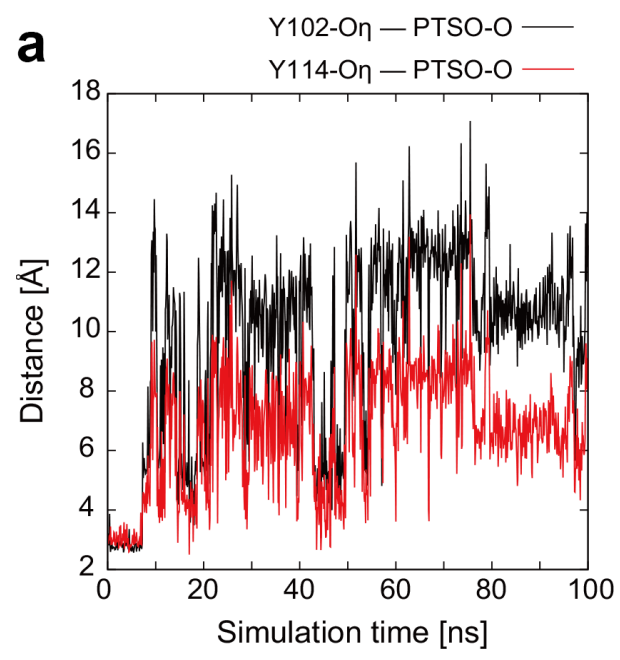

b

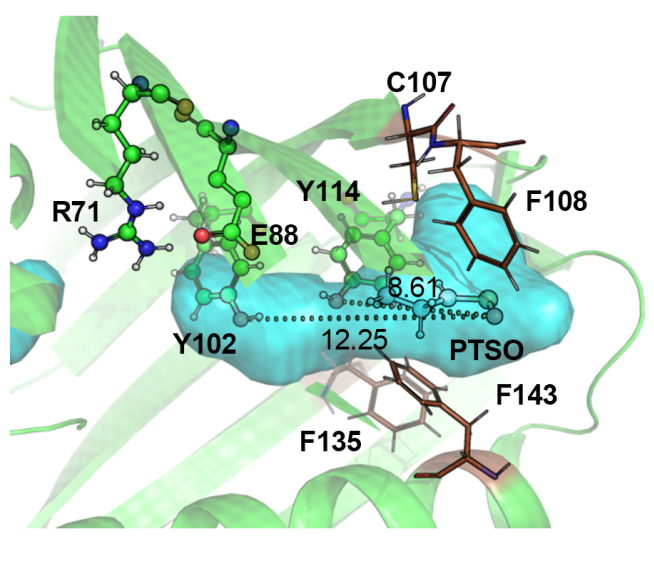

Figure S7. MD simulation of a post-catalysis by AcLFS. (a) Plots of distances in a 100ns trajectory of WT AcLFS in complex with PTSO. (b) A snapshot taken from the MD trajectory at 100 ns. Residues in Gate layer are shown in brown. Space colored in cyan represents the cavity from the Gate to the active site in $A c$ LFS. 


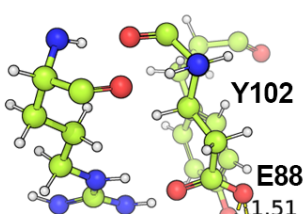

R71 2.75 $33^{\circ}, 1$

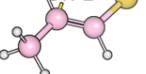

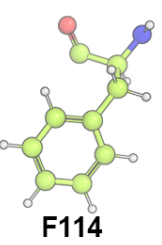

Reactant
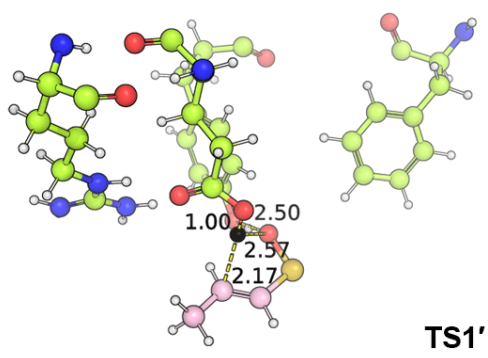

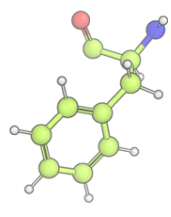

Product

Figure S8. Active site models of the Y114F variant at the stationary points. Optimized structures at the ONIOM(B3LYP/6-31G $+(d, p)$ :AMBER $)$ level of Reactant state (Reactant), Transition state 1' (TS1'), and Product state (Product) are shown. Geometry optimization was started with a snapshot in which the $\mathrm{O}_{1-\mathrm{PSA}}-\mathrm{H} \cdots \mathrm{O}_{\varepsilon 1, \mathrm{E} 88}, \mathrm{O}_{1-\mathrm{PSA}}-\mathrm{H}^{\cdots} \mathrm{O}_{\eta}$, $\mathrm{Y} 102$, and $\mathrm{C}_{2-\mathrm{PSA}} \cdots \mathrm{O}_{\varepsilon 1, \mathrm{E} 88}$ distances were $2.52,2.79$, and $3.65 \AA$, respectively, taken from a MD production run for the Y114F variant. 


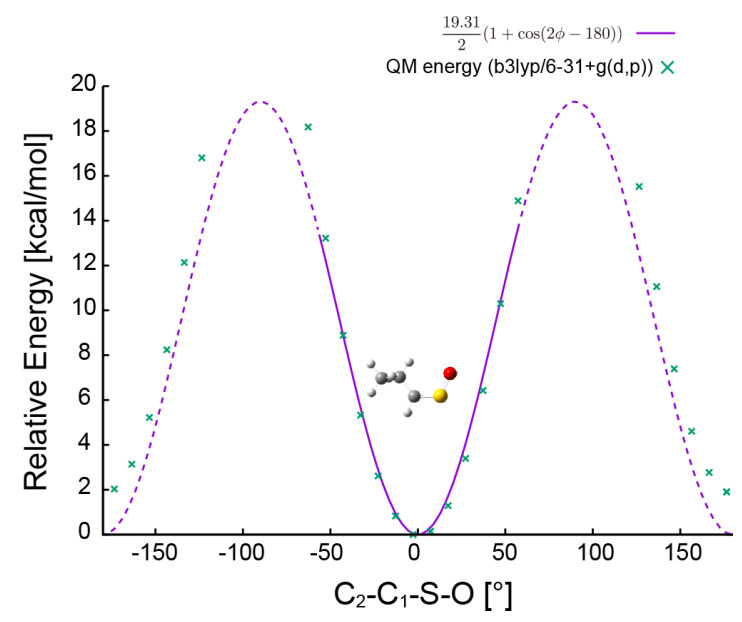

Figure S9. Potential energy scan for a PTSO molecule. QM energies were calculated according to the torsional angles $\mathrm{C}_{2}-\mathrm{C}_{1}-\mathrm{S}-\mathrm{O}$ and at the $\mathrm{B} 3 \mathrm{LYP} / 6-31+\mathrm{G}(\mathrm{d}, \mathrm{p})$ level of theory using Gaussian 16 Rev A.03. The cosine function fitted from the 11 points around $0^{\circ}$ was used for the MD simulations. The additional torsional parameters for PTSO are described in Table S3. 
Table S1. Data collection and refinement statistics

\begin{tabular}{|c|c|c|c|c|}
\hline & Solute-free & + glycerol & $+1,2$-propanediol & + crotyl alcohol \\
\hline \multicolumn{5}{|l|}{ Data collection } \\
\hline Beamline & PF NE3A & PF BL5A & PF NW12A & PF NW12A \\
\hline Wavelength $(\AA)$ & 1.0000 & 1.0000 & 1.0000 & 1.0000 \\
\hline Space group & $P 2_{1} 2_{1} 2_{1}$ & $P 2{ }_{1} 2_{1}{ }_{1}$ & $P 4_{1}$ & $P 4_{3}$ \\
\hline \multicolumn{5}{|l|}{ Cell dimensions } \\
\hline$a, b, c(\AA)$ & $65.1,118.7,124.5$ & $65.0,119.9,125.6$ & $172.9,172.9,65.0$ & $50.1,50.1,141.4$ \\
\hline Molecules per ASU & 4 & 4 & 8 & 2 \\
\hline Resolution $(\AA)$ & $\begin{array}{c}50.00-2.00 \\
(2.07-2.00)^{\mathrm{a}}\end{array}$ & $\begin{array}{l}50.00-1.80 \\
(1.83-1.80)\end{array}$ & $\begin{array}{l}50.00-1.70 \\
(1.76-1.70)\end{array}$ & $\begin{array}{l}50.00-1.80 \\
(1.83-1.80)\end{array}$ \\
\hline Unique reflections & $66,057(6,398)$ & $91,533(8,871)$ & $204,662(16,550)$ & $32,193(1,565)$ \\
\hline Completeness (\%) & $99.6(98.0)$ & $99.7(98.6)$ & $97.3(79.1)$ & $99.9(100.0)$ \\
\hline Multiplicity & $6.7(6.2)$ & $5.8(5.2)$ & $6.1(4.9)$ & $7.4(7.6)$ \\
\hline Mean $I / \sigma I$ & $15.2(2.8)$ & $18.9(2.2)$ & $19.8(4.2)$ & $28.5(2.9)$ \\
\hline$R_{\text {merge }}$ & $0.100(0.676)$ & $0.053(0.600)$ & $0.062(0.378)$ & $0.095(0.532)$ \\
\hline $\mathrm{CC}_{1 / 2}$ & $(0.80)$ & $(0.93)$ & $(0.86)$ & $(0.90)$ \\
\hline \multicolumn{5}{|l|}{ Refinement } \\
\hline Resolution $(\AA)$ & $50.00-2.00$ & $50.00-1.80$ & $50.00-1.70$ & $50.0-1.80$ \\
\hline No. of reflections & 62,884 & 86,948 & 194,424 & 32,099 \\
\hline$R_{\text {work }} / R_{\text {free }}$ & $0.191 / 0.233$ & $0.170 / 0.199$ & $0.230 / 0.260^{\mathrm{b}}$ & $0.182 / 0.209$ \\
\hline No. of atoms & 5,271 & 5,626 & 10,295 & 2,594 \\
\hline Protein & 4,873 & 4,893 & 9,646 & 2,430 \\
\hline Solute & 43 & 85 & 65 & 10 \\
\hline Water & 352 & 648 & 584 & 154 \\
\hline \multicolumn{5}{|l|}{ B-factors } \\
\hline Protein $\left(\AA^{2}\right)$ & 29.7 & 20.3 & 20.1 & 29.3 \\
\hline Solute $\left(\AA^{2}\right)$ & 55.9 & 40.7 & 22.0 & 31.1 \\
\hline Water $\left(\AA^{2}\right)$ & 37.4 & 34.8 & 25.9 & 32.4 \\
\hline \multicolumn{5}{|l|}{ RMS deviation } \\
\hline Bond lengths $(\AA)$ & 0.019 & 0.020 & 0.031 & 0.013 \\
\hline Bond angles $\left({ }^{\circ}\right)$ & 1.87 & 1.93 & 2.83 & 1.70 \\
\hline \multicolumn{5}{|l|}{ Ramachandran plot } \\
\hline Favored (\%) & 99.0 & 98.7 & 95.0 & 99.7 \\
\hline Allowed (\%) & 0.8 & 1.2 & 4.1 & 0.3 \\
\hline Outlier (\%) & 0.17 & 0.17 & 0.85 & 0 \\
\hline PDB ID & 5GTE & $5 \mathrm{GTF}$ & 5GTG & 6IES \\
\hline
\end{tabular}

${ }^{a}$ Values in parentheses are for the highest resolution shell.

${ }^{\mathrm{b}}$ High $R$ factor values because of twinning (see Materials and Methods). 
Table S2. Primer sets used in this study

\begin{tabular}{|c|c|}
\hline \multicolumn{2}{|c|}{ Cloning $^{\text {a }}$} \\
\hline \multicolumn{2}{|c|}{ pET26b- $A c$ LFS } \\
\hline & 5'-GGAATTCCATATGGAGCTAAATCCTGGTGCACC-3' \\
\hline & 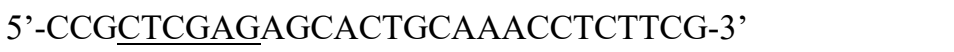 \\
\hline \multicolumn{2}{|c|}{ pPAL7-AcLFS } \\
\hline & 5'-CCCAAGCTTTGGGTGAGCTAAATCCTGGTGCACCTGC-3' \\
\hline & 5'-CGGGATCCTCAAGCACTGCAAACCTCTTCG-3' \\
\hline \multicolumn{2}{|c|}{ Mutagenesis ${ }^{b}$} \\
\hline \multirow[t]{2}{*}{ C107A } & 5'-ACTGAGGCTTTTACAGGGTACGAGGATTAC-3' \\
\hline & 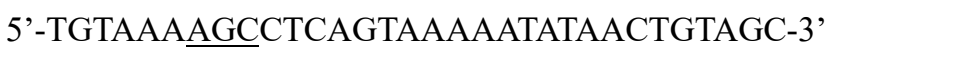 \\
\hline \multirow[t]{2}{*}{ T109A } & 5'-TGTTTTGCAGGGTACGAGGATTACACGGCTAC-3' \\
\hline & 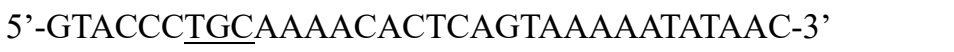 \\
\hline \multirow[t]{2}{*}{ E112Q } & 5'-GGGTACCAGGATTACACGGCTACCATGC-3' \\
\hline & 5'-GTAATCCTGGTACCCTGTAAAACACTCAG-3' \\
\hline \multirow[t]{2}{*}{ M143A } & 5'-GAGGGTGCGACTGAATCTGCATTCACCG-3' \\
\hline & 5'-TTCAGTCGCACCCTCGATATACTTGCACTG-3' \\
\hline \multirow[t]{2}{*}{ L47A } & 5'-ATTAACGCTCACAAGGTCATGCCTTCG-3' \\
\hline & 5'-CTTGTGAGCGTTAATAAAGTCTTTTAGTAGTGTCC-3' \\
\hline \multirow[t]{2}{*}{ M51A } & 5'-AAGGTCGCGCCTTCGTTGTCAGTCTGTG-3' \\
\hline & 5'-CGAAGGCGCGACCTTGTGAAGGTTAATAAAG-3' \\
\hline \multirow[t]{2}{*}{ L54A } & 5'-CCTTCGGCGTCAGTCTGTGAACTGGTAGAAG-3' \\
\hline & 5'-GACTGACGCCGAAGGCATGACCTTGTGAAG-3' \\
\hline \multirow[t]{2}{*}{ V73A } & 5'-CGCTACGCTAAAGGTATAATGCACCCAATAG-3' \\
\hline & 5'-ACCTTTAGCGTAGCGAACACAACCAACAAC-3' \\
\hline \multirow[t]{2}{*}{ M118A } & 5'-GCTACCGCGCAAATAGTGGAGGGTCCTGAG-3' \\
\hline & 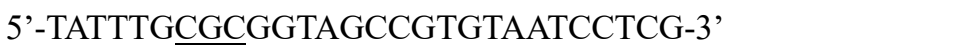 \\
\hline \multirow[t]{2}{*}{ F84A } & 5'-GAGGAAGCTTGGGCCAAGGAGAAGCTGGTG-3' \\
\hline & 5'-GGCCCAAGCTTCCTCTTCTATTGGGTGCATTATAC-3' \\
\hline \multirow[t]{2}{*}{ Y102A } & 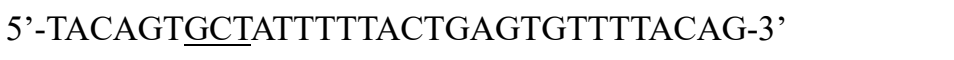 \\
\hline & 5'-AAAAATAGCACTGTAGCTCATGTTCTTATTATCC-3' \\
\hline \multirow[t]{2}{*}{ F104A } & 5'-TATATTGCTACTGAGTGTTTTACAGGGTAC-3' \\
\hline & 5'-CTCAGTAGCAATATAACTGTAGCTCATGTT-3' \\
\hline \multirow[t]{2}{*}{ W133A } & 5'-TTTGACGCGTCTTTTCAGTGCAAGTATATC-3' \\
\hline & 5'-AAAAGACGCGTCAAATCTACTTCCCTTGTG-3' \\
\hline \multirow[t]{2}{*}{ W155A } & 5'-CAGCATGCGGCTACTGAGATAGGTCAGAAAATC-3' \\
\hline & 5’-AGTAGCCGCATGCTGCAGAATCTCGGTGAATG-3' \\
\hline \multirow[t]{2}{*}{ E88A } & 5'-GCCAAGGCGAAGCTGGTGGCGCTGGATAATAAG-3' \\
\hline & 5’-CAGCTTCGCCTTGGCCCAAAATTCCTCTTC-3' \\
\hline \multirow[t]{2}{*}{ R71A } & 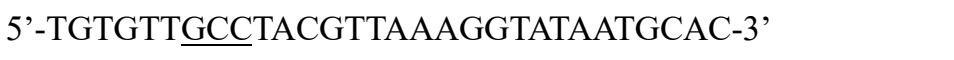 \\
\hline & 5'-AACGTAGGCAACACAACCAACAACATTGGC-3' \\
\hline \multirow[t]{2}{*}{ Y102F } & 5'-TACAGTTTTATTTTTACTGAGTGTTTTACAG-3' \\
\hline & 5'-AAAAATAAAACTGTAGCTCATGTTCTTATTATCC-3' \\
\hline
\end{tabular}




\begin{tabular}{|c|c|}
\hline \multirow[t]{2}{*}{ F104Y } & \\
\hline & 5'-CTCAGTATAAATATAACTGTAGCTCATGTTC-3' \\
\hline \multirow[t]{2}{*}{ Y114F } & 5'-GAGGATTTCACGGCTACCATGCAAATAGTG-3' \\
\hline & 5'-AGCCGTGAAATCCTCGTACCCTGTAAAACAC-3' \\
\hline \multirow[t]{2}{*}{$\mathrm{R} 71 \mathrm{~K}$} & 5'-TGTGTTAAATACGTTAAAGGTATAATGCAC-3' \\
\hline & 5'-AACGTATTTAACACAACCAACAACATTGGC-3' \\
\hline \multirow[t]{2}{*}{ E88Q } & 5'-GCCAAGCAGAAGCTGGTGGCGCTGGATAATAAG-3' \\
\hline & 5'-CAGCTTCTGCTTGGCCCAAAATTCCTCTTC-3' \\
\hline \multirow[t]{2}{*}{ E88D } & 5'-GCCAAGGATAAGCTGGTGGCGCTGGATAATAAG-3' \\
\hline & 5’-CAGCTTATCCTTGGCCCAAAATTCCTCTTC-3' \\
\hline
\end{tabular}

${ }^{\text {a }}$ Underlined sequences correspond to the restriction sites.

$\mathrm{b}$ Triplets underlined correspond to the points of mutation. 


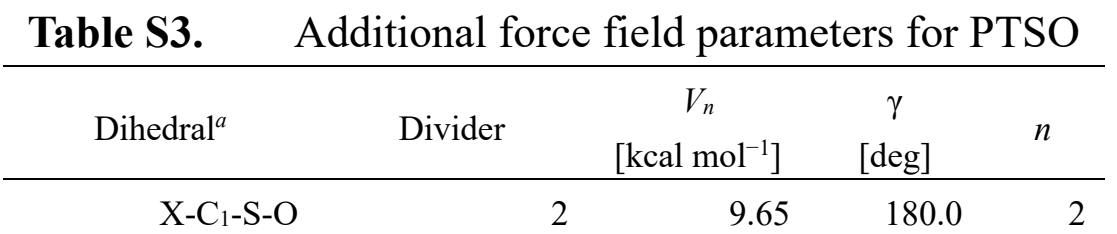

${ }^{a} V_{n}$ is barrier height divided by a factor of $2 ; n$ is periodicity and $\gamma$ is the phase angle for the torsional angle parameters. These parameters were determined by fitting of the optimized energies obtained by single-point energy calculations at the B3LYP/6-31+G(d,p). ' $\mathrm{X}$ ' denotes 'any atom'. 


\section{References}

1. Hiraki, M.; Watanabe, S.; Honda, N.; Yamada, Y.; Matsugaki, N.; Igarashi, N.; Gaponov, Y.; Wakatsuki, S. High-Throughput Operation of SampleExchange Robots with Double Tongs at the Photon Factory Beamlines. Journal of Synchrotron Radiation 2008, 15, 300-303.

2. Kabsch, W. XDS. Acta Crystallogr D Biol Crystallogr 2010, 66, 125-132. 3. Adams, P. D.; Afonine, P. V.; Bunkóczi, G.; Chen, V. B.; Davis, I. W.; Echols, N.; Headd, J. J.; Hung, L.-W.; Kapral, G. J.; Grosse-Kunstleve, R. W.; McCoy, A. J.; Moriarty, N. W.; Oeffner, R.; Read, R. J.; Richardson, D. C.; Richardson, J. S.; Terwilliger, T. C.; Zwart, P. H. PHENIX: a Comprehensive Python-Based System for Macromolecular Structure Solution. Acta Crystallogr D Biol Crystallogr 2010, 66, 213-221.

4. Emsley, P.; Lohkamp, B.; Scott, W. G.; Cowtan, K. Features and Development of Coot. Acta Crystallogr D Biol Crystallogr 2010, 66, 486-501.

5. Vagin, A.; Teplyakov, A. An Approach to Multi-Copy Search in Molecular Replacement. Acta Crystallogr D Biol Crystallogr 2000, 56, 1622-1624. 6. Murshudov, G. N.; Skubak, P.; Lebedev, A. A.; Pannu, N. S.; Steiner, R. A.; Nicholls, R. A.; Winn, M. D.; Long, F.; Vagin, A. A. REFMAC5 for the Refinement of Macromolecular Crystal Structures. Acta Crystallogr D Biol Crystallogr 2011, 67, 355-367.

7. Winn, M. D.; Ballard, C. C.; Cowtan, K. D.; Dodson, E. J.; Emsley, P.; Evans, P. R.; Keegan, R. M.; Krissinel, E. B.; Leslie, A. G. W.; McCoy, A.; McNicholas, S. J.; Murshudov, G. N.; Pannu, N. S.; Potterton, E. A.; Powell, H. R.; Read, R. J.; Vagin, A.; Wilson, K. S. Overview of the CCP4 Suite and Current Developments. Acta Crystallogr D Biol Crystallogr 2011, 67, 235-242.

8. Baker, N. A.; Sept, D.; Joseph, S.; Holst, M. J.; McCammon, J. A. Electrostatics of Nanosystems: Application to Microtubules and the Ribosome. Proc Natl Acad Sci U S A 2001, 98, 10037-10041.

9. Masamura, N.; Ohashi, W.; Tsuge, N.; Imai, S.; Ishii-Nakamura, A.; Hirota, H.; Nagata, T.; Kumagai, H. Identification of Amino Acid Residues Essential for Onion Lachrymatory Factor Synthase Activity. Biosci Biotechnol Biochem 2012, 76, 447-453. 
10. Jorgensen, W. L.; Chandrasekhar, J.; Madura, J. D.; Impey, R. W.; Klein, M. L. Comparison of Simple Potential Functions for Simulating Liquid Water. The Journal of Chemical Physics 1983, 79, 926-935.

11. Case, D. A.; Cheatham, T. E. r.; Darden, T.; Gohlke, H.; Luo, R.; Merz, K. M. J.; Onufriev, A.; Simmerling, C.; Wang, B.; Woods, R. J. The Amber Biomolecular Simulation Programs. J Comput Chem 2005, 26, 1668-1688.

12. Wang, J.; Wolf, R. M.; Caldwell, J. W.; Kollman, P. A.; Case, D. A. Development and Testing of a General Amber Force Field. J Comput Chem 2004, $25,1157-1174$.

13. Wang, J.; Wang, W.; Kollman, P. A.; Case, D. A. Automatic Atom Type and Bond Type Perception in Molecular Mechanical Calculations. J Mol Graph Model 2006, 25, 247-260.

14. Block, E.; Gillies, J.Z.; Gillies, C. W.; Bazzi, A. A.; Putman, D.; Revelle, L. K.; Wang, D.; Zhang, X. Allium Chemistry: Microwave Spectroscopic Identification, Mechanism of Formation, Synthesis, and Reactions of (E,Z)Propanethial S-Oxide, the Lachrymatory Factor of the Onion (Allium cepa). $J$ Am Chem Soc 1996, 118, 7492-7501.

15. Darden, T.; York, D.; Pedersen, L. Particle Mesh Ewald: An N $\log (\mathrm{N})$ Method for Ewald Sums in Large Systems. The Journal of Chemical Physics 1993, 98, 10089-10092.

16. Bussi, G.; Donadio, D.; Parrinello, M., Canonical Sampling through Velocity Rescaling. J Chem Phys 2007, 126, 014101.

17. Maseras, F.; Morokuma, K. IMOMM: A New Integrated ab initio + Molecular Mechanics Geometry Optimization Scheme of Equilibrium Structures and Transition States. Journal of Computational Chemistry 1995, 16, 1170-1179. 18. Becke, A. D. Density-Functional Thermochemistry. III. The Role of Exact Exchange. The Journal of Chemical Physics 1993, 98, 5648-5652.

19. Lee, C.; Yang, W.; Parr, R. G. Development of the Colle-Salvetti Correlation-Energy Formula into a Functional of the Electron Density. Phys. Rev. B 1988, 37, 785-789.

20. Vreven, T.; Byun, K. S.; Komáromi, I.; Dapprich, S.; Montgomery, J. A.; Morokuma, K.; Frisch, M. J. Combining Quantum Mechanics Methods with Molecular Mechanics Methods in ONIOM. J Chem Theory Comput 2006, 2, 815- 
826.

S26 